\title{
Co-evolution of black holes and galaxies: the role of selection biases
}

\author{
Laura Portinari \\ Tuorla Observatory, University of Turku, Väisäläntie 20, FIN-21500 Piikkiö, Finland
}

\begin{abstract}
Quasars are tracers of the cosmological evolution of the Black Hole mass - host galaxy relation, and indicate that the formation of $\mathrm{BH}$ anticipated that of the host galaxies. We find that selection effects and statistical biases dominate the interpretation of the observational results; and co-evolution (= constant $\mathrm{BH} /$ galaxy mass ratio) is still compatible with observations.
\end{abstract}

Keywords. Galaxies: active, high-redshift, formation, evolution; quasars: general

In the local Universe, the mass of the central SuperMassive Black Hole (SMBH) correlates with that of the host galaxy with a typical mass ratio $\Gamma \equiv M_{B H} / M_{\star} \simeq 0.002$. At high redshifts the $\Gamma$ ratio can be estimated in QSOs, by measuring the SMBH mass via the "virial technique", and determining the host galaxy luminosity (and stellar mass) from deep imaging, after subtracting the (dominant) QSO point-source.

QSO host studies indicate that $\Gamma$ increases with redshift, and was 5-10 times larger than now by $\mathrm{z}=3$ : SMBH appear to have formed before the bulk of their host galaxies (Peng et al. 2006; Decarli et al. 2010; Merloni et al. 2010). However, a proper interpretation of the data must consider a number of selection biases and observational errors.

1. Lauer bias The intrinsic relation between SMBH and host mass has a scatter (about $0.3 \mathrm{dex}$ ) and is weighted by the galaxy luminosity function: a very massive $\mathrm{BH}$ is more likely to be an outlier in the relation, hosted in a relatively low-mass galaxy and with high $\Gamma$, than belong to a very massive host, as these are rare. A QSO host is a typical median host galaxy behind a selected quasar/SMBH mass; as high $z$ QSOs are very massive $\mathrm{BH}\left(10^{9}-10^{10} \mathrm{M}_{\odot}\right)$, they will tendentially trace higher-than-average $\Gamma$ values creating an apparent evolution (Lauer et al. 2007); see Fig. 1a,b.

2. Overluminosity bias of QSO hosts. Nuclear activity at high $\mathrm{z}$ is supposed to be associated with recent mergers and starbursts, making QSO hosts overluminous with respect to the general galaxy population for the same stellar (and BH) mass (Fig. 1c). This induces an overestimate of the stellar mass $M_{\star}$, and an underestimate of the $\Gamma$ ratio. This bias acts in the opposite direction to the Lauer bias.

3. Shen-Kelly bias is a Malmquist bias on BH masses, as high redshift QSOs trace the most massive, rare BH. The typical error on measured BH masses of 0.4 dex artificially boosts them, by statistically overestimating the mass of smaller, more common BH (Shen \& Kelly 2010); see Fig. 1d.

In Portinari et al. (2012), we implemented all these biases when comparing QSO host data to the publicly available semi-analytical model (SAM) of De Lucia \& Blaizot (2007). Their model predicts a negligible evolution of the "intrinsic" $\mathrm{BH}$-host relation (i.e. $\Gamma$ varies little with redshift). Yet, once all the biases are included, the model can account for the observed strong variation of $\Gamma$, and co-evolution (constant intrinsic $\Gamma$ ) is still compatible with the data (Fig. 1). The model however has difficulty in predicting the number of the most massive $\mathrm{BH}\left(>10^{9} \mathrm{M}_{\odot}\right)$ at high $z$. 

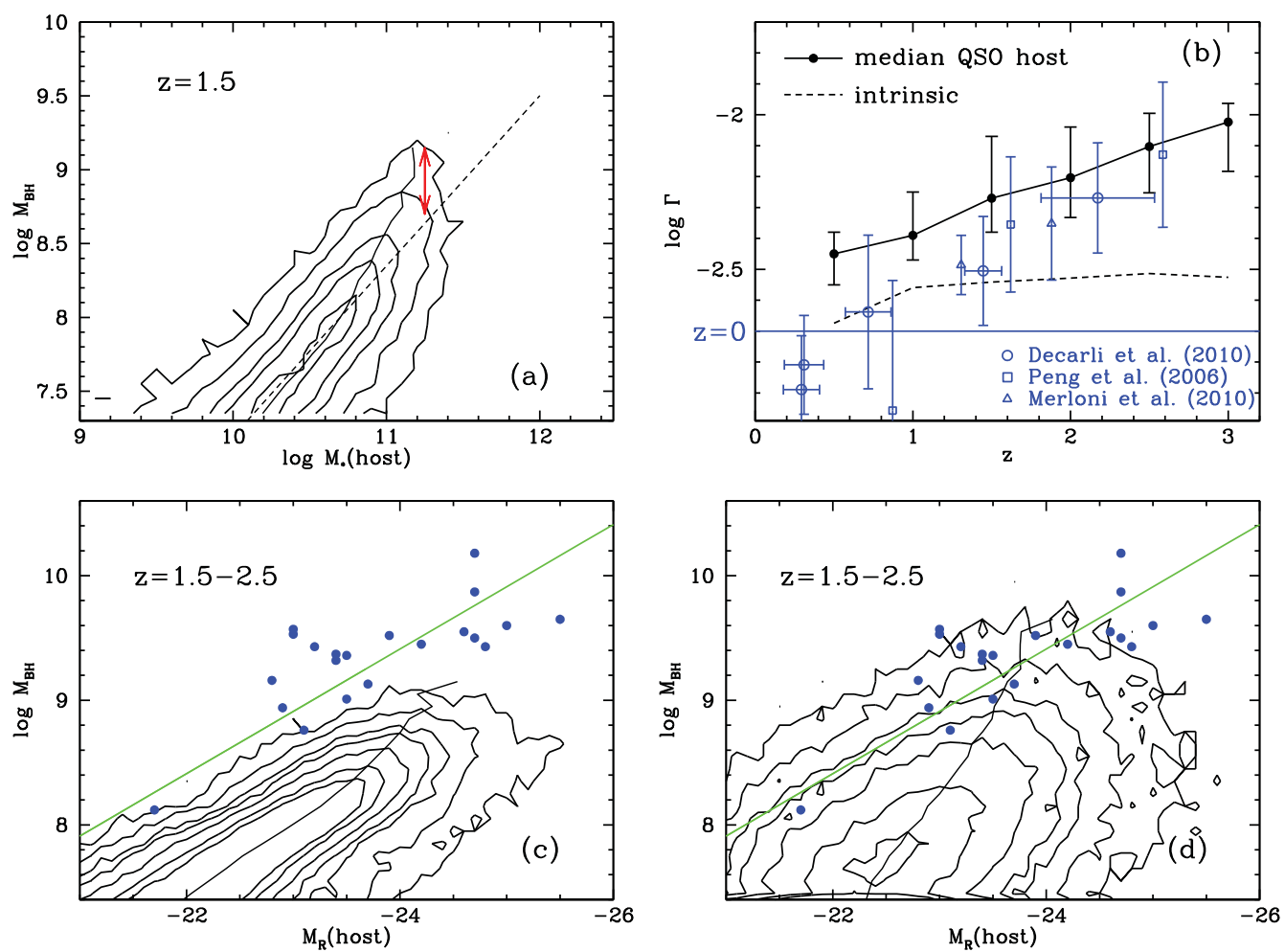

Figure 1. (a) Contour plot of the $\left(M_{B H}, M_{\star}\right)$ distribution of high $z$ galaxies from the SAM of De Lucia \& Blaizot (2007). The dashed line marks the "intrinsic" $M_{B H}-M_{\star}$ relation, the solid line traces the "median" QSO host at given $M_{B H}$. The difference (updown arrow) translates into a spurious $\Gamma$ evolution (Lauer bias). (b) While the intrinsic $\Gamma$ ratio evolves little (dashed line) the median QSO hosts (full dots connected by solid line) trace a strong apparent evolution, comparable to the observed one (open symbols). (c) In the observational ( $M_{B H}$, luminosity) plane, model QSO hosts are overluminous and offset from the observed data (full dots). (d) After convolving with the observational error $M_{B H}$, the theoretical distribution is in fair agreement with observations (Shen-Kelly bias); although it is missing the most massive BH.

The aforementioned biases are due to the intrinsic scatter in the BH-host relations, combined with the fact that QSOs sample the massive end of the $\mathrm{BH}$ and galaxy mass function. These biases dominate the interpretation of the observational results, as they mimic, and are hardly distinguishable from, evolution. We should turn to relatively low redshift QSO hosts, where evolution is negligible, to better understand the scatter and biases in the real Universe, disentangled from evolution. The Stripe 82 sample of almost 400 QSO hosts at $z<0.5$ by Falomo et al. (2014) will be useful to that purpose.

\section{References}

De Lucia, G. \& Blaizot, J., 2007, MNRAS 375, 1

Decarli, R., Falomo, R., Treves, A., Labita, M., Kotilainen, J., \& Scarpa, R., 2010, MNRAS 402, 2453

Falomo, R., Bettoni, D., Karhunen, K., Kotilainen, J. K., \& Uslenghi, M., 2014, MNRAS 440, 476

Lauer, T. R., Tremain, S., Richstone, D., \& Faber, S. M., 2007, ApJ 670, 249

Merloni, A., Bongiorno, A., Bolzonella, M., et al. 2010, ApJ 708, 37

Peng, C. Y., Impey, C. D., Rix, H.-W., et al. 2006, ApJ 649, 616

Portinari, L., Kotilainen, J., Falomo, R., \& Decarli, R., 2012, MNRAS 420, 732

Shen, Y. \& Kelly, B. C., 2010, ApJ 713, 41 